\title{
Facilitation of Chemotaxis Activity of Mesenchymal Stem Cells via Stromal Cell-Derived Factor-1 and Its Receptor May Promote Ectopic Ossification of Human Spinal Ligaments
}

\author{
Shunfu Chin, Ken-Ichi Furukawa, Keigo Kurotaki, Shunpei Nagasaki, Kanichiro Wada, \\ Gentaro Kumagai, Shigeru Motomura, and Yasuyuki Ishibashi \\ Departments of Orthopaedic Surgery (S.C., K.W., G.K., Y.I.) and Pharmacology (K.-I.F., K.K., S.N., S.M.), Graduate School of \\ Medicine, Hirosaki University, Hirosaki, Aomori, Japan
}

Received October 16, 2018; accepted January 18, 2019

\begin{abstract}
Mesenchymal stem cells (MSCs) have been used to elucidate the pathogenesis of numerous diseases. Our recent study showed that MSCs may conduce to the ossification of spinal ligaments. Stromal cell-derived factor-1 (SDF-1) and CXC chemokine receptor 4 (CXCR4) regulate MSC migration. Moreover, their expression is elevated in sites of damage and remodeling in pathologic states. We explored the possible role of the SDF1/CXCR4 axis in the chemotactic behavior of MSCs in the ossification of spinal ligaments. Specimens of thoracic vertebra ossified ligamentum flavum (OLF) and non-OLF plaques were received from patients in whom we had performed spine surgery. Paraffin-embedded tissue sections were prepared for immunohistochemical staining. Cultured MSCs from the ligamentum flavum were prepared for in vitro analyses. We observed SDF-1 and CXCR4 localization immunohistochemically in the perivascular
\end{abstract}

area and collagenous matrix of ligaments and in chondrocytes near the ossification front of OLF. And then, immunohistochemical staining showed a close relationship between MSCs and the SDF-1/CXCR4 axis. In the in vitro analyses, expression of the SDF-1/CXCR4 and the migratory capacity of MSCs in OLF were remarkably higher compared with non-OLF MSCs. Furthermore, the migration of MSCs was upregulated by SDF-1 and downregulated by treatment with AMD3100 $\left(\mathrm{C}_{28} \mathrm{H}_{54} \mathrm{~N}_{8} .8 \mathrm{HCl}\right)$, a specific antagonist for CXCR4. All in vitro test data showed a significant difference in MSCs from OLF compared with non-OLF MSCs. Our results reveal that the SDF-1/CXCR4 axis may contribute to an MSC-mediated increase in the ossification process, indicating that the SDF-1/CXCR4 axis may become a potential target for a novel therapeutic strategy for ossification of spinal ligaments.

\section{Introduction}

Ectopic ossification occurs in human ligaments of spine, resulting in spinal canal narrowing and eventually evoking spinal cord injury and myelopathy in patients experiencing various degrees of neurologic symptoms. In severe cases, surgery may be required even if the surgery is also associated with difficult situations such as a higher risk of neurologic complications (Choi et al., 2005). Therefore, the development of a safe and effective pharmacotherapy to control ectopic ossification is necessary. Up to the present, the cause of the ectopic ossification of spinal ligaments has been proved in a widespread way and has been connected to a variety of factors such as epidemiology, genetics, metabolism, and mechanics (Furukawa, 2006). However, pharmaceuticals having

This work was supported by a Grant-in-Aid from the Practical Research Project for Rare/Intractable Diseases from the Japan Agency for Medical Research and Development (AMED) and a Grant-in-Aid from the Ministry of Education, Culture, Sports, Science and Technology of Japan [Grant 17K10916]. No potential conflicts of interest relevant to this article are reported.

https://doi.org/10.1124/jpet.118.254367. effectiveness for ossification therapy and/or advanced suppression is still not clear.

Mesenchymal stem cells (MSCs) have been found in several tissues, including muscle, bone marrow, adipose tissue, synovium, cartilage, and ligament. The isolated cells were used not only for tissue repair and regeneration, but also to make clear the pathogenesis of various diseases such as vascular calcification and calcifying tendinopathy (Segawa et al., 2009; Yee Lui et al., 2011). Our previous study (Chin et al., 2013) showed that ossified ligamentum flavum (OLF) has remarkable accumulation of MSCs compared with nonOLF MSCs (control), and that chondrocytes around the ossification front in OLF express MSC markers. Furthermore, an enhancement in the osteogenic differentiation potentiality has been found in MSCs from ossified ligament samples. This leaning toward the osteogenic lineage may constitute causality in the ossification (Harada et al., 2014; Chiba et al., 2015). Based on these findings, MSCs are thought to be involved in the process of ectopic ossification occurring in spinal ligaments.

Stromal cell-derived factor-1 (SDF-1) and its receptor CXC chemokine receptor 4 (CXCR4) are expressed in several

ABBREVIATIONS: CXCR4, CXC chemokine receptor 4; FCS, fetal calf serum; MSC, mesenchymal stem cell; OLF, ossified ligamentum flavum; $\mathrm{PCR}$, polymerase chain reaction; SDF-1, stromal cell-derived factor-1. 
tissues, and the SDF-1/CXCR4 axis is related to a range of physiologic processes such as neovascularization and MSC migration and cytokine secretion (Mirshahi et al., 2000; Petit et al., 2007). Overexpression of SDF-1 and CXCR4 has been found in sites of active damage and remodeling in pathologic states (Zgraggen et al., 2014), and a specific CXCR4 antagonist, AMD3100 $\left(\mathrm{C}_{28} \mathrm{H}_{54} \mathrm{~N}_{8} .8 \mathrm{HCl}\right)$, is used in clinical pathology. At this point, we hypothesized that the SDF-1/CXCR4 axis might have an important influence in ectopic ossification of spinal ligaments. One possibility is that the SDF-1/CXCR4 axis regulates the chemotactic behavior of MSCs in ossified ligaments, resulting in MSC migration and ossification. These findings would allow great insight into the target site for medical treatment.

Therefore, we investigated the expression of both SDF-1 and CXCR4 in spinal ligaments and the potential effect of the SDF1/CXCR4 axis on migration by treatment with SDF-1 and AMD3100 in MSCs from OLF and non-OLF. The results we obtained revealed a significant role of the SDF-1/CXCR4 axis in the accumulation of MSCs in ossified ligaments, and that the modulation of the SDF-1/CXCR4 axis may be a novel strategy for the treatment of the ossification of spinal ligaments.

\section{Materials and Methods}

Spinal Ligament Samples. Spinal ligament samples of the ligamentum flavum (LF) were collected en bloc from 12 patients who underwent surgery at the thoracic vertebral level. Samples of OLF were obtained from six patients (four males and two females; mean age at surgery, 69 years; age range, $56-77$ years) who had posterior decompression surgery for OLF. As a control, non-OLF samples were obtained from six patients (five males and one female; mean age, 50 years; age range, 22-81 years) who had undergone posterior surgery for spinal tumor, syringomyelia, or burst fracture. No patients had evidence of congenital osteoarthropathy or rheumatism. The study protocol was approved by the Human Ethics Review Committee of Hirosaki University Hospital, and sufficient informed consent was obtained from each patient.

Cell Isolation and Culture. We isolated MSCs as described previously (Harada et al., 2014). Cells were identified as MSCs by fluorescence-activated flow cytometry. MSCs were stained using an anti-human CD34 mouse antibody coupled with phycoerythrin and an anti-human CD105 mouse antibody coupled with PerCP-Cy5.5, respectively (BD Biosciences, San Jose, CA). Cells at first passage $(1 \times$ $10^{6}$ ) were harvested and washed with PBS containing $20 \mu \mathrm{l} / \mathrm{ml}$ fetal calf serum (FCS). Cells suspended in PBS $(50 \mu \mathrm{l})$ containing specific antibodies were incubated for 45 minutes on ice. The cells were washed with PBS, filtered through a $35-\mu \mathrm{m}$ strainer (BD Biosciences, Bedford, MA), suspended in PBS (1 ml), and analyzed by using a FACSAria II Flow Cytometer (BD Biosciences). For cell sorting, gates were defined as negative for CD34 and positive for CD105 according to the intensity of the fluorescence from the isotype control. Data were analyzed by using BD FACSDiva software version 6.1.3 (BD Biosciences).

Preparation of Tissue. Non-OLF and OLF were fixed with formaldehyde $(100 \mathrm{mg} / \mathrm{ml})$ at $4^{\circ} \mathrm{C}$ for 1 week. Samples of ossified LF were further decalcified with KC-X solution (Falma, Tokyo, Japan) for about 4 days. Then, serial $4-\mu \mathrm{m}$-thick sections were prepared using paraffin-embedded specimens and processed for $\mathbf{H} \& \mathbf{E}$ staining and immunohistochemistry.

Immunohistochemical Staining. Immunohistochemical analysis was accomplished as described previously (Chin et al., 2013). Briefly, antigen retrieval and blocking of non-specific protein binding were performed after deparaffinization of sections. Then, the sections were incubated overnight at $4^{\circ} \mathrm{C}$ with a single primary antibody or a mixture of two primary antibodies diluted in $10 \mathrm{mg} / \mathrm{ml}$ BSA. Primary antibodies against MSC markers included a mouse monoclonal anti-CD73 antibody (1:50 dilution; Abcam, Cambridge, MA), rabbit polyclonal anti-CD90 antibody (1:100 dilution; Abcam), and mouse monoclonal anti-CD105 antibody (1:50 dilution; Dako, Tokyo, Japan). A mouse monoclonal anti-SDF-1 antibody (1:50 dilution; Abcam); rabbit polyclonal anti-SDF-1 antibody (1:50 dilution; Abcam); mouse monoclonal anti-CXCR4 antibody (1:100 dilution; Abcam), and rabbit polyclonal anti-CXCR4 antibody (1:500 dilution; Abcam) were used to detect the chemokine SDF-1 and its receptor, CXCR4.

For single staining, the sections were treated with secondary and tertiary agents, and then $N, N^{\prime}$-diaminobenzidine was used to visualize peroxidase deposition at antigenic sites. Finally, the sections were lightly counterstained with H\&E. And then, we calculated the prevalence of SDF-1-positive and CXCR4-positive cells in the collagenous matrix area. The ratio of positive cells to nucleated cells was the defined prevalence.

For double staining, sections were incubated with antibodies with green and red-fluorescent dyes, and finally the sections were lightly counterstained with DAPI (4',6'-diamidino-2-phenylindole dihydrochloride). After staining with double immunofluorescence, the sections were observed under a confocal laser-scanning microscope (Digital Eclipse C1si/C1 plus; Nikon Instruments, Tokyo, Japan). Image data were saved as raw TIFF files without further thresholding or filtering (e.g., without background subtraction).

CXCR4 Expression Analysis by Using Real-Time Polymerase Chain Reaction. Total RNA was extracted from cultured MSCs using an RNeasy Mini Kit (QIAGEN, Valencia, CA) according to the manufacturer instructions. Genomic DNA contamination was eliminated by DNase treatment. First-strand cDNA was synthesized from total RNA $(1 \mu \mathrm{g})$ using a standard random hexamer priming technique. Real-time polymerase chain reaction (PCR) was carried out with Power SYBR Green PCR Master Mix on an ABI Prism 7000 Sequence Detection System (Applied Biosystems, Foster City, CA). Specific primer pairs for CXCR4 (GenBank accession number NM_003467; forward primer, 5'-CTGCCCTCCTGCTGACTA-3'; reverse primer, 5' -GGGTAGAAGCGGTCACAGA-3') were designed by using Primer-BLAST software (http://www.ncbi.nlm.nih.gov/tools/ primer-blast/). Conditions for PCRs were as follows: $95^{\circ} \mathrm{C}$ for 1 minute, and then 50 cycles of $95^{\circ} \mathrm{C}$ for 15 seconds and $60^{\circ} \mathrm{C}$ for 1 minute. All samples were analyzed in parallel for glycerol 3-phosphate dehydrogenase (G3PDH) expression as an internal standard. The data of realtime PCR experiments were represented as $\mathrm{Ct}$ values. The $\mathrm{Ct}$ value is defined as the cycle number at which the amount of PCR product exceeds the threshold level. We used the comparative $\mathrm{Ct}$ method to compare mRNA expression in samples with that in the control in each experiment. Reactions were carried out in triplicate for each cell sample. Results are expressed as the mean \pm S.D. $(n=6)$. Graphs depict relative expression levels compared with the control (non-OLF MSCs), unless otherwise indicated.

CXCR4 Expression Analysis by Western Blotting. After MSCs from non-OLF and OLF patients (six cell samples each) reached confluency, the cells were lysed in RIPA buffer (250 mM Tris-HCL, $\mathrm{pH}$ 7.6, $150 \mathrm{mM} \mathrm{NaCl}, 10 \mathrm{mg} / \mathrm{ml} \mathrm{NP} 40,10 \mathrm{mg} / \mathrm{ml} \mathrm{Na}$-deoxycholate, and $10 \mathrm{mg} / \mathrm{ml} \mathrm{SDS})$. After a Bradford protein assay, protein samples (3 $\mu \mathrm{g}$ each) were resolved by SDS-PAGE and then transferred to polyvinylidene difluoride membranes (Immobilon-FL; Millipore Corporation, Billerica, MA). The membranes were blocked with $1 \mathrm{mg} / \mathrm{ml}$ dry skim milk in PBS with $1 \mu \mathrm{l} / \mathrm{ml}$ Tween 20 and incubated with primary antibodies against CXCR4 (rabbit polyclonal antibody, 1:500 dilution; Abcam) or $\beta$-actin (rabbit polyclonal antibody, 1:1000 dilution; Abcam) and then an Alexa Fluor 680-labeled goat anti-rabbit secondary antibody (1:1000; Abcam). Proteins were detected using the Odyssey Imaging System (LI-COR Biosciences, Lincoln, NE).

Cell Migration Analysis. Cell migration was assayed by the scratch wound healing method using an in vitro incisional wound model, as reported previously (Liang et al., 2007). MSCs were cultured 
A

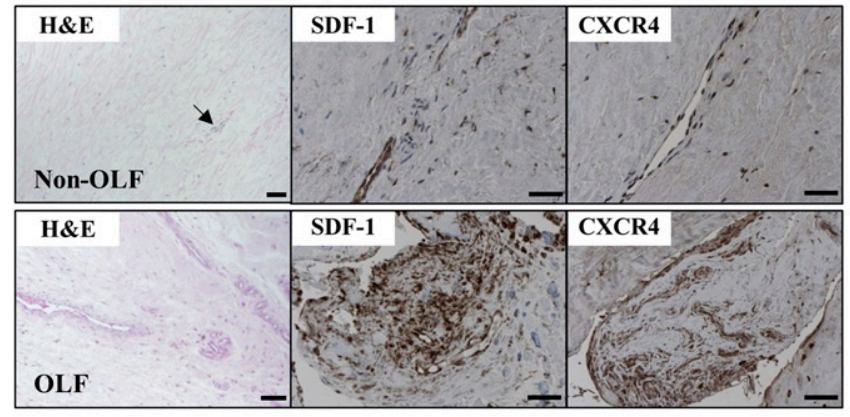

B

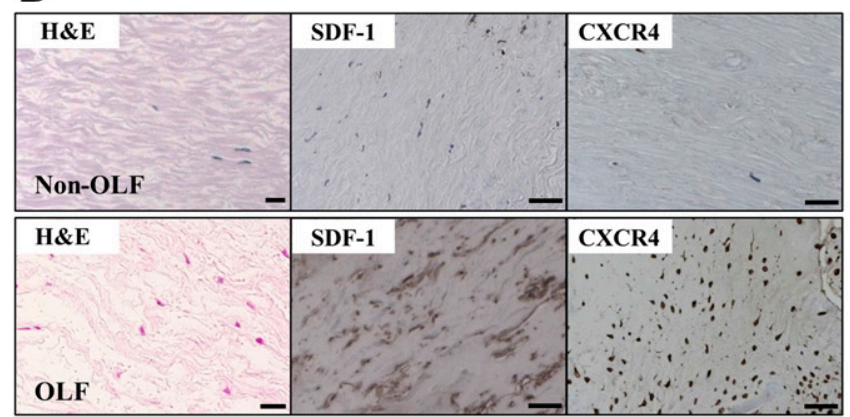

C

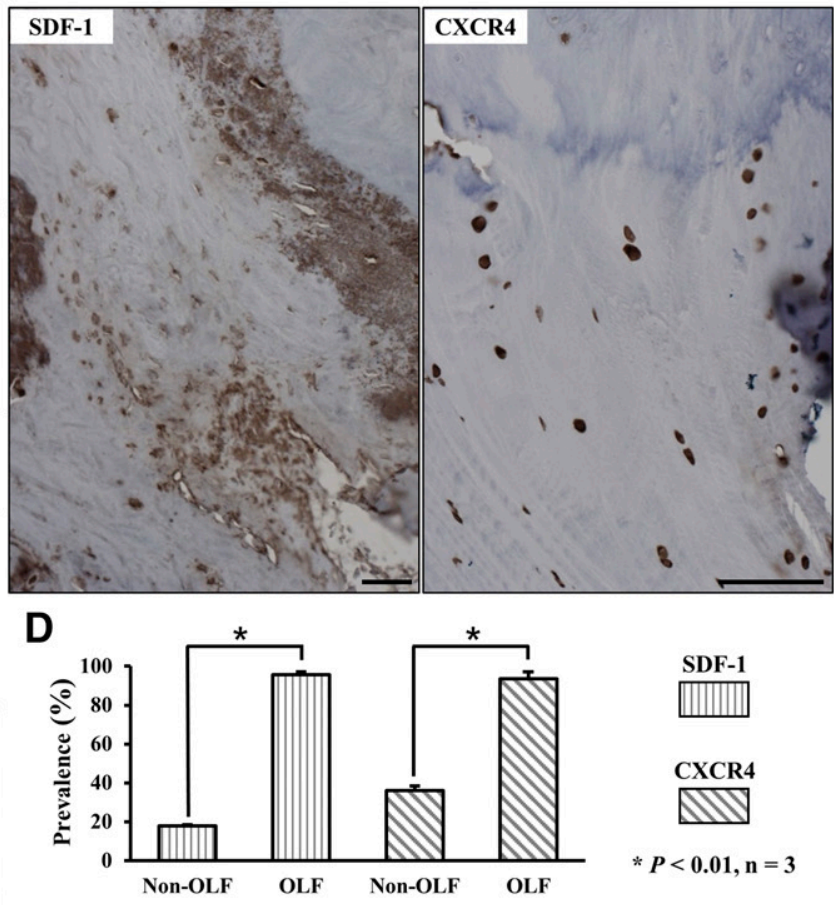

Fig. 1. SDF-1 and CXCR4-positive cells in spinal ligaments. Non-OLF and OLF in the blood vessel region (arrow: blood vessel) (A) and collagenous matrix (B). (C) The ossification front in OLF. (D) The prevalence in collagenous matrix from three samples per group. ${ }^{*} P<0.01$ compared with the control (non-OLF group). Scale bar, $50 \mu \mathrm{m}$.

in six-well plates (BD Biosciences, Franklin Lakes, NJ) with $\alpha$-minimal essential medium containing $100 \mu \mathrm{l} / \mathrm{ml} \mathrm{FCS.} \mathrm{At} 80 \%$ confluence, FCS in the medium was reduced to $20 \mu \mathrm{l} / \mathrm{ml}$ to avoid cell proliferation during the assay period. After 1 day of incubation, scratch wounds of $1 \mathrm{~mm}$ in width were created in confluent monolayers by using an aseptic 1000- $\mu$ l pipette tip. After detached cells were washed out, the wounded monolayers were cultured for 24 hours. Images of the wound area were obtained at 0 and 24 hours using a phase contrast microscope (Olympus, Tokyo, Japan). The area between the wound edges was analyzed using NIH ImageJ software (Bethesda, MD). The wound area was defined as follows: wound healing $(\%)=[1-$ (wound area $(t=24$ hours $) /$ wound area $(t=0$ hour $))] \times 100$. Data were averaged over six independent experiments. Each experiment was repeated three times. MSCs were treated with CXCR4 inhibitor AMD3100 (Abcam) at a final concentration $300 \mathrm{nM}$ to determine the dependency of migration on CXCR4.

Cell Chemotaxis Assay. Chemotactic migration was evaluated, as described previously (Mori et al., 2012), in chemotaxis chambers (Chemotaxicell; Kurabo, Osaka, Japan) inserted into a 24-well culture plate. MSCs $\left(2 \times 10^{4}\right)$ were spread into the upper chamber, and the lower chamber was filled with PBS with or without SDF-1 at various dosages $(10,30$, and $100 \mathrm{ng} / \mathrm{ml})$ in $\alpha$-minimal essential medium containing $20 \mu \mathrm{l} / \mathrm{ml} \mathrm{FCS}$. The cells were incubated for 48 hours at $37^{\circ} \mathrm{C}$ with $5 \% \mathrm{CO}_{2}$. The chambers were washed two times with PBS and then fixed with $100 \mathrm{mg} / \mathrm{ml}$ formaldehyde for 5 minutes, followed by washing two times with water. The chamber was stained with methyl green for 20 minutes and washed three times with water, and then the number of stained cells in the filter, which had migrated from the upper chamber, was counted under a microscope.

Statistical Analysis. All data analyses were performed by KyPlot 5.0 software (Kyenslab, Tokyo, Japan). Differences among groups were analyzed using one-way analysis of variance with the StudentNewman-Keuls post hoc correction procedure. $P$ values less than 0.05 were regarded as statistically significant.

\section{Results}

Localization of SDF-1 and CXCR4 in Spinal Ligament Tissues. SDF-1 and CXCR4 distributions in spinal ligaments were analyzed immunohistochemically. Figure 1 shows representative images. Single immunostaining showed SDF-1 expression and both SDF-1-positive and CXCR4-positive cells around blood vessels in both OLF and non-OLF (control) tissue samples. In non-OLF samples, little expression of SDF1 and a small number of SDF-1 and CXCR4-positive cells were observed poorly in the perivascular area (Fig. 1A). Conversely, in OLF tissue samples, there was abundant angiogenesis, and high expression of SDF-1 and great numbers of SDF-1-positive and CXCR4-positive cells were observed around blood vessels (Fig. 1A). Next, we examined SDF-1-positive and CXCR4 expression within the collagenous matrix. In control non-OLF tissues, a small number of fibroblast-like cells were observed in the collagenous fibers with regular arrangement. Moreover, only a few fibroblast-like cells were positive for SDF-1 and CXCR4. Conversely, numerous fibroblast-like cells were positive for SDF-1 and CXCR4 in OLF tissues. In addition, the expression of SDF-1 was observed at a very low level in control non-OLF tissue compared with its strong expression in OLF tissue among the irregularly arranged and fragmented collagenous fibers (Fig. 1, B and D). In OLF tissue samples, we observed intense expression of SDF-1 and a large number of chondrocyte-like cells expressing both SDF-1 and CXCR4 around the ossification front (Fig. 1C).

Expression Change of the SDF-1/CXCR4 Axis in MSCs. Because high expression of SDF-1 and CXCR4 was observed in OLF tissues, which was similar to our previously described expression change in MSCs (Chin et al., 2013), we 
A

OLF : collagenous matrix
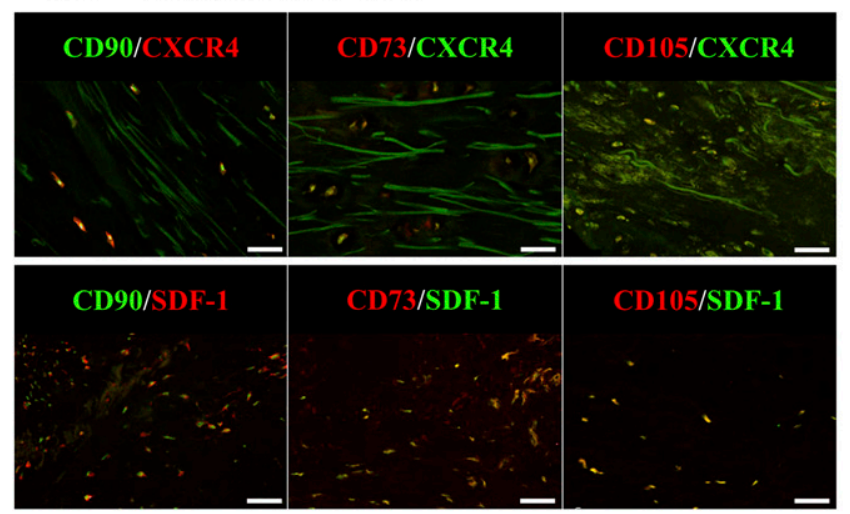

B

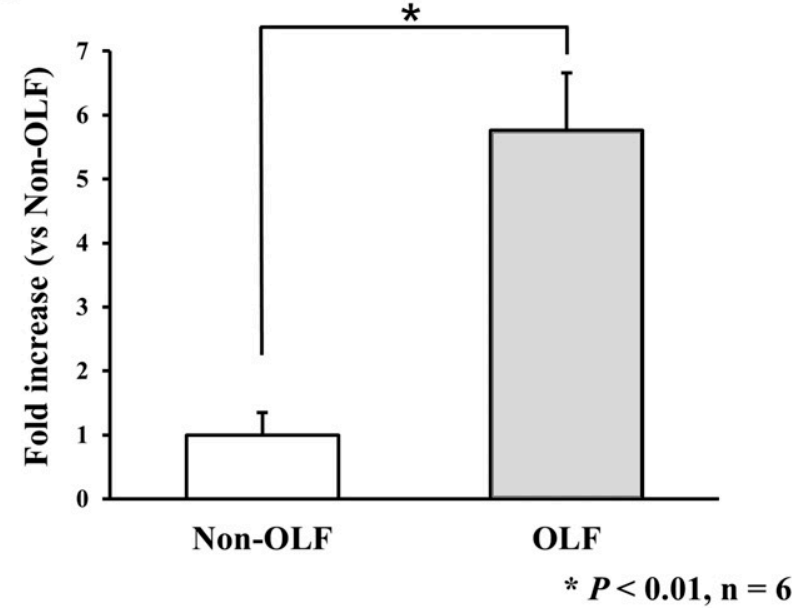

OLF : ossification front
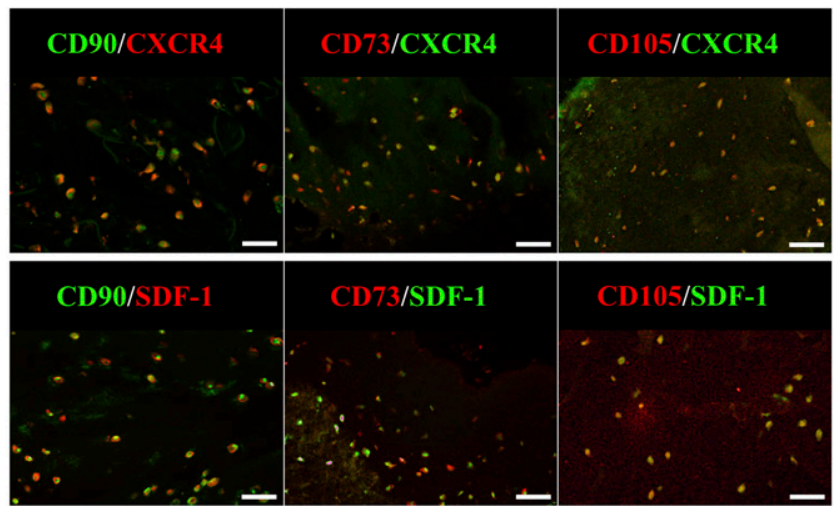

C

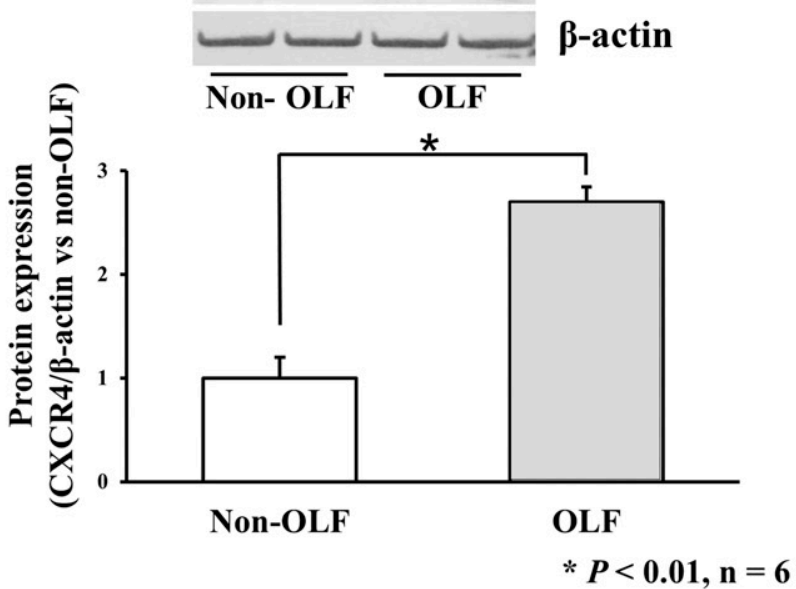

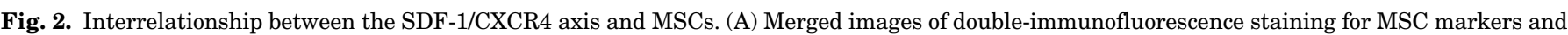

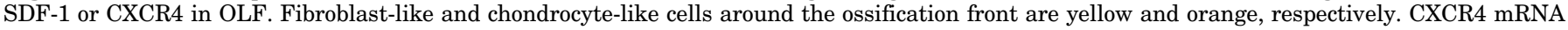

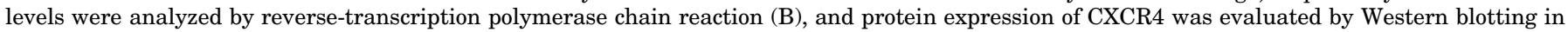

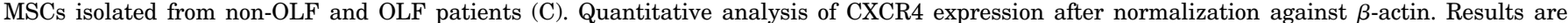

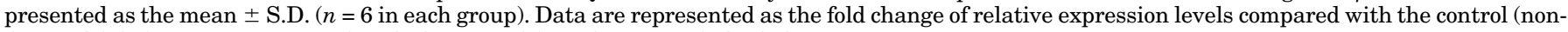
OLF MSCs). $* P<0.01$ compared with the control (non-OLF group). Scale bar, $50 \mu \mathrm{m}$.

investigated the relationship between SDF-1-positive or CXCR4-positive cells and MSCs, which are identified by the expression of CD73, CD90, and CD105, in collagenous matrix and the ossification front of OLF samples. Double-immunofluorescence staining was performed for SDF-1/CD73, SDF-1/CD90, SDF-1/CD105, CXCR4/CD73, CXCR4/CD90, and CXCR4/CD105. The results showed colocalization of SDF-1-positive or CXCR4-positive cells and MSC marker-positive cells (Fig. 2A). To confirm these discoveries, we assayed the CXCR4 mRNA levels by quantitative real-time PCR in the non-OLF and OLF groups. CXCR4 mRNA was extremely expressed in MSCs from OLF samples, but it was expressed at a very low level in MSCs from non-OLF samples (Fig. 2B). Statistical analysis indicated a substantial increase in CXCR4 expression of MSCs derived from OLF than from non-OLF samples $(P<$ $0.01)$. Protein expression analysis showed a similar result (Fig. 2C).

Chemotaxis Activity Assay of the SDF-1/CXCR4 Axis in MSCs. We evaluated the migratory capacity of MSCs derived from both non-OLF and OLF groups by a scratch wound assay. After 24 hours of culture in the medium containing $20 \mu \mathrm{l} / \mathrm{ml}$ FCS to avoid cell proliferation, MSCs from OLF samples migrated into $>45 \%$ of the scratched area, whereas MSCs from non-OLF samples migrated into $<18 \%$ of the scratched area. It showed a significant difference between these samples $(P<0.01)$ (Fig. 3A). To find out the effect of activating the SDF-1/CXCR4 pathway on the regulation of MSC migration in vitro, we first determined the regulatory role of CXCR4 in MSC migration by the scratch wound assay. The migratory activity of MSCs derived from the non-OLF group pretreated with AMD3100 showed a decrease in comparison with that of MSCs without AMD3100 pretreatment. Furthermore, MSCs derived from the OLF group showed a significant degradation of migratory activity in response to AMD3100 $(P<0.01)$ (Fig. 3B). Next, we examined whether SDF-1 pretreatment influenced the chemotaxis of MSCs toward the SDF-1 gradient in a transwell migration analysis. After PBS or SDF-1 treatment for 24 hours, the total number of MSCs that migrated through the porous membranes of transwell chambers was obviously higher in the OLF group than in the non-OLF group in all treatments $(P<0.01)$. The higher migration ability of MSCs 
A
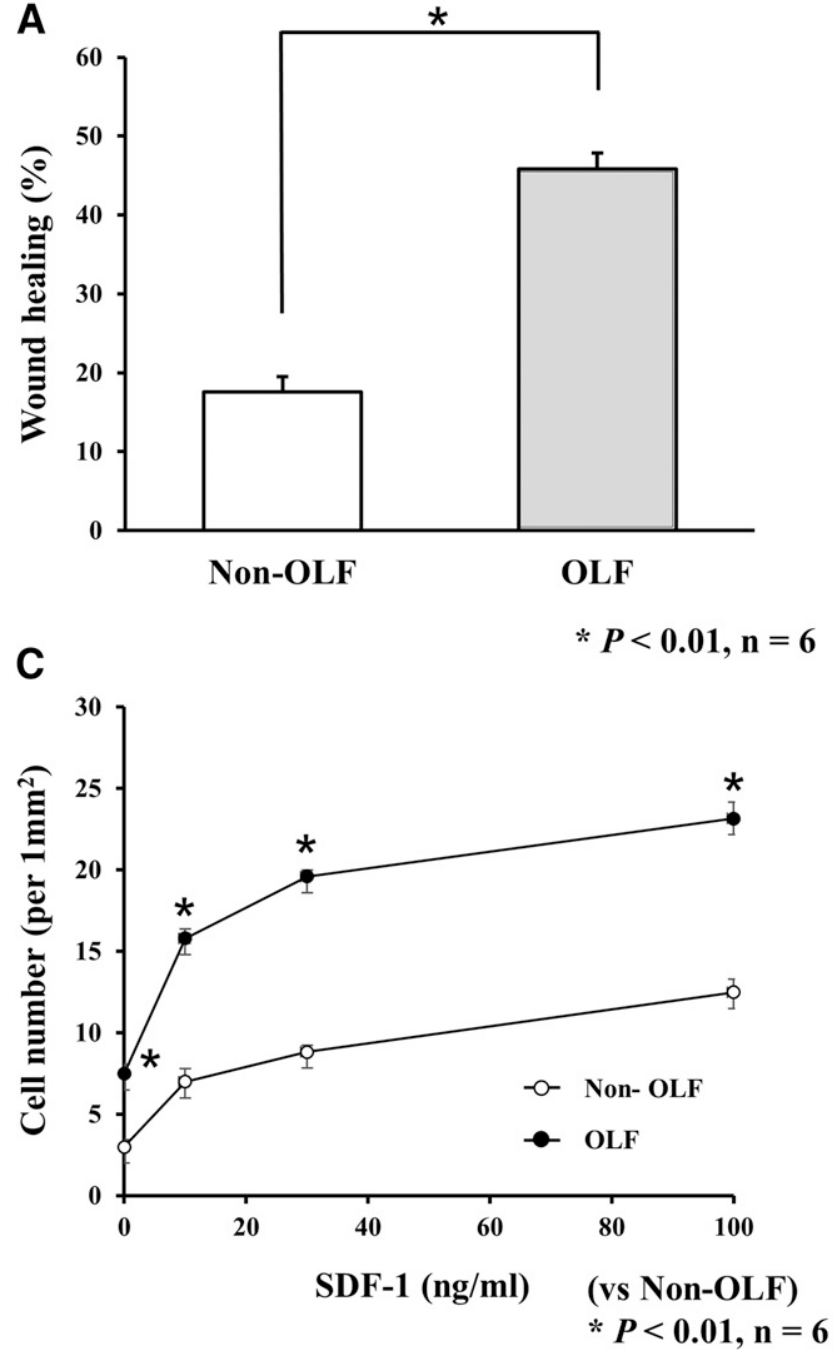

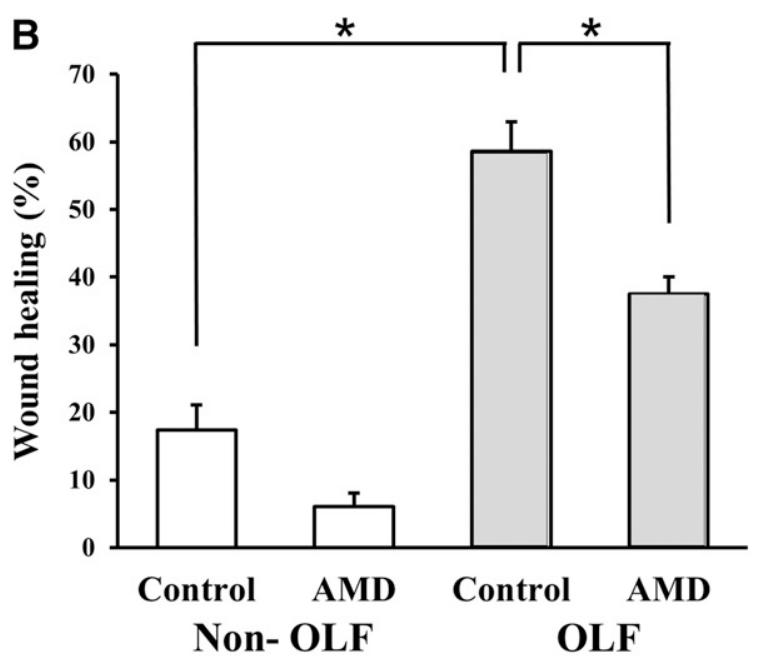

$300 \mathrm{nM}$ AMD3100

$* P<0.01, \mathrm{n}=6$

Fig. 3. Chemotaxis activity assay of the SDF-1/CXCR4 axis in MSCs. (A) The migration activity of MSCs was analyzed by a wound healing assay. Results are presented as the mean \pm S.D. of percentages of the wound healing size from six independent experiments in both non-OLF and OLF groups. $* P<0.01$, compared with the control (non-OLF group). (B) MSCs were pretreated with AMD3100 (300 nM) and then analyzed by the wound healing assay to determine the dependency of migration on CXCR4. Results are presented as the mean \pm S.D. of percentages of the wound healing size from six independent experiments in non-OLF AMD3100-untreated, non-OLF AMD3100-treated, OLF AMD3100-untreated, and OLF AMD3100-treated groups. The percentages of the wound healing size in the AMD3100-treated samples were compared with those in the untreated control. (C) MSC migration toward SDF-1 was measured in a chemotaxis chamber containing various concentrations of SDF-1 $(0,10,30$, and $100 \mathrm{ng} / \mathrm{ml})$. After incubation, transmigrated MSCs were collected and counted. Results are presented as the mean \pm S.D. from six independent experiments in both the non-OLF and OLF groups. The number of cells per square millimeter was calculated.

from the OLF group was consistent with the results of the scratch wound assay. Additionally, $10 \mathrm{ng} / \mathrm{ml}$ SDF-1 pretreatment resulted in an approximately 2 -fold increase in the number of migrated MSCs compared with the PBS control. In addition, 30 and $100 \mathrm{ng} / \mathrm{ml}$ SDF-1 pretreatment resulted in an increase of the MSC migration ability in response to SDF-1 treatment in a dose-dependent manner in both the non-OLF and OLF groups (Fig. 3C).

\section{Discussion}

Immunodetection has identified the SDF-1/CXCR4 axis, which resides in both collagenous matrix and small blood vessels, in multiple organs in vivo (Zgraggen et al., 2014; Liu et al., 2016). However, there was no report describing the detailed expression in human spinal ligaments in situ. Here, we show the existence of the SDF-1/CXCR4 axis in both
non-OLF and OLF in vivo. Furthermore, we observed a high expression in ossified spinal ligaments from OLF patients, which is similar to the expression change in MSCs observed previously (Chin et al., 2013). Fibroblast-like cells in the collagenous matrix and chondrocyte-like cells around the ossification front, which may be derived from MSCs and contribute to ectopic ossification through endochondral ossification and aggregation of abnormal fibrocartilage or cartilaginous cells (Yee Lui et al., 2011; Chin et al., 2013), expressed SDF-1 and CXCR4. Furthermore, double immunostaining showed the coexistence of SDF-1/CXCR4 and MSC markers. Real-time PCR and Western blot analyses showed that the expression level of CXCR4 in MSCs from OLF was significantly higher than that in MSCs from non-OLF. These results suggest a close relationship between MSCs and the SDF1/CXCR4 axis, and that they are committed to the presence/ development of ectopic ossification. 


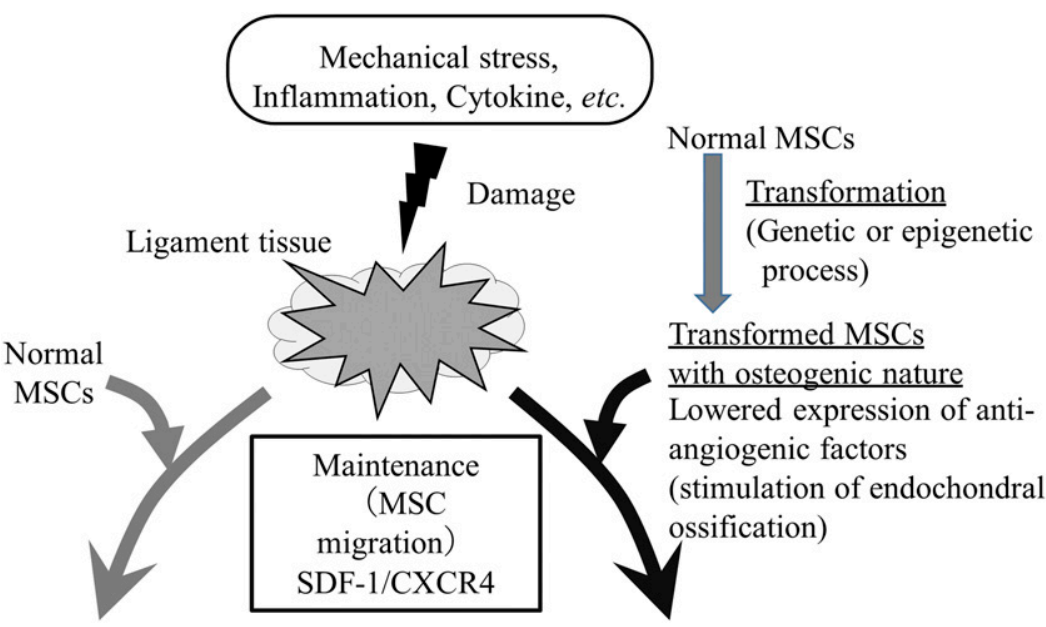

Tissue repair
Ectopic ossification of ligament
Fig. 4. Possible role of SDF-1/CXCR4 in ectopic ossification of spinal ligaments.
The present results showed a large amount of vascularization and high expression of the chemokine SDF-1 and its receptor CXCR4 in OLF tissues. In addition, MSCs derived from OLF showed an increased migration ability in response to SDF-1. Vascular activation is also observed in chronic inflammatory diseases (Baluk et al., 2005), and various proinflammatory chemokines have been reported to exhibit angiogenic properties (Kiefer and Siekmann, 2011; Zgraggen et al., 2014). Particularly, the chemokine SDF-1 and its receptor CXCR-4 have been found to have an angiogenic activity (Pablos et al., 1999; Mirshahi et al., 2000). Elevated expression of the SDF-1/CXCR4 axis and vascularization have been found in lesion sites. Blood vessels are thought to be a place of origin of MSCs, providing conclusive proof that neovascularization is related to heterotopy calcification of tissues (Lounev et al., 2009; Bautch, 2011). In our previous study (Chin et al., 2013), MSCs were not only in the perivascular area, but also were diffused over ligament tissue in OLF, suggesting that MSC migration plays an important part in the ligament ossification process. Recently, it has been reported that the SDF-1/CXCR4 axis is an important regulator of stem cell migration and homing (Kokovay et al., 2010; Liu et al., 2016), and MSCs synthesize and secrete multiple paracrine factors that influence their own migration (Boomsma and Geenen, 2012; Liang et al., 2012). Therefore, we suspect that SDF-1 is secreted from MSCs as a division of the repairing process caused by mechanical stress that leads to collagenous fiber rupture in spinal ligaments. In addition, we consider that the SDF-1/CXCR4 axis plays an important part in the vascularization and accumulation of MSCs in neoplastic blood vessels and collagenous matrix through their migration.

MSCs are suspected to play an important part in the ectopic ossification process of OLF (Chin et al., 2013), and the SDF$1 /$ CXCR4 axis is involved in a range of physiologic processes such as endochondral bone development (Murata et al., 2012; Toupadakis et al., 2012). Moreover, an interesting study (Otsuru et al., 2008) has proved that circulating bone marrow-derived osteoblast progenitor cells are recruited to the bone-forming site in a bone-forming model via the SDF1/CXCR4 axis. Although the relationship between MSCs and the SDF-1/CXCR4 axis in ectopic ossification remains controversial, these reports and our results suggest that MSCs may be related to the ectopic ossification of OLF through upregulation of the SDF-1/CXCR4 axis. The regulatory mechanism of MSCs and the SDF-1/CXCR4 axis in ectopic ossification should be pursued further in the future.

Increased expression of the SDF-1/CXCR4 axis has been reported in various diseases such as colitis and arthritis, and these studies indicate that the SDF-1/CXCR4 axis is involved in disease maintenance and progression (De Klerck et al., 2005; Mikami et al., 2008). Indeed, the inhibition of CXCR4 exerts anti-inflammatory effectiveness in various inflammatory diseases (Lukacs et al., 2002). Furthermore, the experiment on animals showed the therapeutic effect of psoriasis (a chronic inflammatory skin disease) after treatment with the CXCR4 antagonist AMD3100, which is associated with decreased inflammatory vascularization and cell accumulation (Zgraggen et al., 2014). In the current study, we found that inhibition of the SDF-1/CXCR4 axis signaling by AMD3100 significantly abolished the migration ability of MSCs in the OLF group, whereas no remarkable decrease was detected in the non-OLF group. These observations indicate that one of the noteworthy findings is that blocking CXCR4 is directly related to the reduced migration ability of MSCs derived from OLF samples. According to the above results, we suggest that the SDF-1/CXCR4 axis may serve as an up-to-date therapeutic target for the ossification of spinal ligaments in reducing angiogenesis and MSC accumulation. Future studies using, for example, the ossification of spinal ligaments in an animal model to investigate the in vivo ossification suppressant effect of the CXCR4 antagonist AMD3100 may elucidate the role of the SDF-1/CXCR4 axis and its relationship with MSCs in the ectopic ossification process of OLF.

Figure 4 depicts a possible role of SDF-1/CXCR4 in ectopic ossification of spinal ligaments.

The authentic role of MSCs is in the repair of tissue that has been damaged by, for example, mechanical stress or inflammation (Maxson et al., 2012). The MSCs are promoted by SDF1/CXCR4 axis and migrate into the damaged area of the ligament, resulting in the recovery of the ligament. This process occurs in normal tissues. However, in OLF tissue 
samples from patients, the osteogenic nature of MSCs has become transformed through genetic or epigenetic change (Chiba et al., 2015). MSCs promoted into the damaged area evoked ossification of the tissue instead of repair. Furthermore, if the transformed MSCs have lost an antiangiogenic ability (unpublished data), then MSCs permit vascular invasion and facilitate ossification of ligament through endochondral ossification process (Yamamoto et al., 2002; Shi et al., 2016). This idea is supported by a clinical observation in which blood loss after surgery in patients with ossified spinal ligaments was significantly higher than that in patients without ossification (Kishiya et al., 2009). This observation suggests an increase in angiogenesis in ossified spinal ligaments.

The first limitation of this study is the absence of sufficient data characterizing MSCs in the tissue sections. Confirmation of the CD73, CD90, and CD105 surface markers is required because MSCs have no unique antibody. In the future, a four-color staining procedure with CD73, CD90, CD105, and SDF-1 or CXCR4 will supply a more accurate representation of the relationship between MSCs and the SDF-1/CXCR4 axis. Second, AMD3100 only induced about a $50 \%$ decrease in wound healing in both OLF and non-OLF samples. Because there are other chemokine/chemokine receptor axes in addition to the SDF-1/CXCR4 axis in the recruitment of MSCs, the potential roles of other chemokine receptors expressed by MSCs, such as transforming growth factor $\beta$ receptors (Labour et al., 2016), should be considered in future studies.

In conclusion, our study showed that expression of the SDF1/CXCR4 axis in human spinal ligaments was higher in OLF, and that chondrocytes that exist in the ossification front were positive for SDF-1 and CXCR4. The migratory capacity of MSCs derived from OLF was remarkably higher than that of MSCs derived from non-OLF, which was upregulated by SDF1 treatment in a dose-dependent manner. Additionally, the migration activity of MSCs was downregulated by pretreatment with a CXCR4 inhibitor, particularly MSCs from OLF. These observations may suggest a role of the SDF-1/CXCR4 axis in MSC migration during the pathogenesis of OLF. We suspect that the SDF-1/CXCR4 axis plays an important role in the ectopic ossification process of OLF.

\section{Acknowledgments}

We thank Dr. Kazumasa Ueyama, Dr. Naoki Echigoya, Dr. Toru Yokoyama, Dr. Atsushi Ono, Dr. Takashi Tomita, Dr. Takuya Numasawa, and Hiroki Mizukami for contributions to sample provision and valuable comments.

\section{Authorship Contributions}

Participated in research design: Chin, Furukawa, Wada, Kumagai, Motomura, and Ishibashi.

Conducted experiments: Chin, Furukawa, Kurotaki, and Nagasaki.

Performed data analysis: Chin, Furukawa, Kurotaki, and Nagasaki.

Wrote or contributed to the manuscript writing and editing: Chin, Furukawa, Wada, Kumagai, Motomura, and Ishibashi.

\section{References}

Baluk P, Tammela T, Ator E, Lyubynska N, Achen MG, Hicklin DJ, Jeltsch M, Petrova TV, Pytowski B, Stacker SA, et al. (2005) Pathogenesis of persistent lymphatic vessel hyperplasia in chronic airway inflammation. $J$ Clin Invest 115 : 247-257.

Bautch VL (2011) Stem cells and the vasculature. Nat Med 17:1437-1443.
Boomsma RA and Geenen DL (2012) Mesenchymal stem cells secrete multiple cytokines that promote angiogenesis and have contrasting effects on chemotaxis and apoptosis. PLoS One 7:e35685.

Chiba N, Furukawa K, Takayama S, Asari T, Chin S, Harada Y, Kumagai G, Wada $\mathrm{K}$, Tanaka T, Ono A, et al. (2015) Decreased DNA methylation in the promoter region of the WNT5A and GDNF genes may promote the osteogenicity of mesenchymal stem cells from patients with ossified spinal ligaments. J Pharmacol Sci 127:467-473.

Chin S, Furukawa K, Ono A, Asari T, Harada Y, Wada K, Tanaka T, Inaba W, Mizukami H, Motomura S, et al. (2013) Immunohistochemical localization of mesenchymal stem cells in ossified human spinal ligaments. Biochem Biophys Res Commun 436:698-704

Choi S, Lee SH, Lee JY, Choi WG, Choi WC, Choi G, Jung B, and Lee SC (2005) Factors affecting prognosis of patients who underwent corpectomy and fusion for treatment of cervical ossification of the posterior longitudinal ligament: analysis of 47 patients. J Spinal Disord Tech 18:309-314.

De Klerck B, Geboes L, Hatse S, Kelchtermans H, Meyvis Y, Vermeire K, Bridger G, Billiau A, Schols D, and Matthys P (2005) Pro-inflammatory properties of stromal cell-derived factor-1 (CXCL12) in collagen-induced arthritis. Arthritis Res Ther 7: R1208-R1220.

Furukawa K (2006) Current topics in pharmacological research on bone metabolism: molecular basis of ectopic bone formation induced by mechanical stress. J Pharmacol Sci 100:201-204.

Harada Y, Furukawa K, Asari T, Chin S, Ono A, Tanaka T, Mizukami H, Murakami M, Yagihashi S, Motomura S, et al. (2014) Osteogenic lineage commitment of mesenchymal stem cells from patients with ossification of the posterior longitudinal ligament. Biochem Biophys Res Commun 443:1014-1020.

Kiefer F and Siekmann AF (2011) The role of chemokines and their receptors in angiogenesis. Cell Mol Life Sci 68:2811-2830.

Kishiya M, Furukawa K, Yokoyama T, Kudo H, Ono A, Numasawa T, Wada K and Toh S (2009) Comparison of cardiovascular parameters between patients with ossification of posterior longitudinal ligament and patients with cervical spondylotic myelopathy. J Spinal Disord Tech 22:361-366.

Kokovay E, Goderie S, Wang Y, Lotz S, Lin G, Sun Y, Roysam B, Shen Q, and Temple S (2010) Adult SVZ lineage cells home to and leave the vascular niche via differential responses to SDF1/CXCR4 signaling. Cell Stem Cell 7 163-173.

Labour MN, Riffault M, Christensen ST, and Hoey DA (2016) TGF $\beta 1$ - induced recruitment of human bone mesenchymal stem cells is mediated by the primary cilium in a SMAD3-dependent manner. Sci Rep 6:35542.

Liang CC, Park AY, and Guan JL (2007) In vitro scratch assay: a convenient and inexpensive method for analysis of cell migration in vitro. Nat Protoc 2: $329-333$

Liang J, Huang W, Yu X, Ashraf A, Wary KK, Xu M, Millard RW, Ashraf M, and Wang Y (2012) Suicide gene reveals the myocardial neovascularization role of mesenchymal stem cells overexpressing CXCR4 (MSC(CXCR4)). PLoS One 7: e46158.

Liu MH, Bian BS, Cui X, Liu LT, Liu H, Huang B, Cui YH, Bian XW, and Zhou Y (2016) Mesenchymal stem cells regulate mechanical properties of human degenerated nucleus pulposus cells through SDF-1/CXCR4/AKT axis. Biochim Biophys Acta 1863:1961-1968.

Lounev VY, Ramachandran R, Wosczyna MN, Yamamoto M, Maidment AD, Shore EM, Glaser DL, Goldhamer DJ, and Kaplan FS (2009) Identification of progenitor cells that contribute to heterotopic skeletogenesis. J Bone Joint Surg Am 91:652-663.

Lukacs NW, Berlin A, Schols D, Skerlj RT, and Bridger GJ (2002) AMD3100, a CxCR4 antagonist, attenuates allergic lung inflammation and airway hyperreactivity. Am J Pathol 160:1353-1360.

Maxson S, Lopez EA, Yoo D, Danilkovitch-Miagkova A, and Leroux MA (2012) Concise review: role of mesenchymal stem cells in wound repair. Stem Cells Transl Med 1:142-149.

Mikami S, Nakase H, Yamamoto S, Takeda Y, Yoshino T, Kasahara K, Ueno S, Uza N, Oishi S, Fujii N, et al. (2008) Blockade of CXCL12/CXCR4 axis ameliorates murine experimental colitis. J Pharmacol Exp Ther 327:383-392.

Mirshahi F, Pourtau J, Li H, Muraine M, Trochon V, Legrand E, Vannier J, Soria J, Vasse M, and Soria C (2000) SDF-1 activity on microvascular endothelial cells: consequences on angiogenesis in in vitro and in vivo models. Thromb Res 99: $587-594$

Mori Y, Yamaguchi M, Terao Y, Hamada S, Ooshima T, and Kawabata S (2012) $\alpha$-Enolase of Streptococcus pneumoniae induces formation of neutrophil extracellular traps. J Biol Chem 287:10472-10481.

Murata K, Kitaori T, Oishi S, Watanabe N, Yoshitomi H, Tanida S, Ishikawa M, Kasahara T, Shibuya H, Fujii N, et al. (2012) Stromal cell-derived factor 1 regulates the actin organization of chondrocytes and chondrocyte hypertrophy. PLoS One 7:e37163.

Otsuru S, Tamai K, Yamazaki T, Yoshikawa H, and Kaneda Y (2008) Circulating bone marrow-derived osteoblast progenitor cells are recruited to the boneforming site by the CXCR4/stromal cell-derived factor-1 pathway. Stem Cells $\mathbf{2 6}$ $223-234$.

Pablos JL, Amara A, Bouloc A, Santiago B, Caruz A, Galindo M, Delaunay T, Virelizier JL, and Arenzana-Seisdedos F (1999) Stromal-cell derived factor is expressed by dendritic cells and endothelium in human skin. Am J Pathol 155: 1577-1586.

Petit I, Jin D, and Rafii S (2007) The SDF-1-CXCR4 signaling pathway: a molecular hub modulating neo-angiogenesis. Trends Immunol 28:299-307.

Segawa Y, Muneta T, Makino H, Nimura A, Mochizuki T, Ju YJ, Ezura Y, Umezawa A, and Sekiya I (2009) Mesenchymal stem cells derived from synovium, meniscus, anterior cruciate ligament, and articular chondrocytes share similar gene expression profiles. J Orthop Res 27:435-441. 
Shi L, Cai G, Shi J, Guo Y, Chen D, Chen D, and Yang H (2016) Ossification of the posterior ligament is mediated by osterix via inhibition of the $\beta$-catenin signaling pathway. Exp Cell Res 349:53-59.

Toupadakis CA, Wong A, Genetos DC, Chung DJ, Murugesh D, Anderson MJ, Loots GG, Christiansen BA, Kapatkin AS, and Yellowley CE (2012) Long-term administration of AMD3100, an antagonist of SDF-1/CXCR4 signaling, alters fracture repair. J Orthop Res 30:1853-1859.

Yamamoto Y, Furukawa K, Ueyama K, Nakanishi T, Takigawa M, and Harata S (2002) Possible roles of CTGF/Hcs24 in the initiation and development of ossification of the posterior longitudinal ligament. Spine 27:1852-1857.
Yee Lui PP, Wong YM, Rui YF, Lee YW, Chan LS, and Chan KM (2011) Expression of chondro-osteogenic BMPs in ossified failed tendon healing model of tendinopathy. J Orthop Res 29:816-821.

Zgraggen S, Huggenberger R, Kerl K, and Detmar M (2014) An important role of the SDF-1/CXCR4 axis in chronic skin inflammation. PLoS One 9:e93665.

Address correspondence to: Ken-Ichi Furukawa, Department of Pharmacology, Graduate School of Medicine, Hirosaki University, 5 Zaifu-cho, Hirosaki, Aomori 036-8562, Japan. E-mail: furukawa@hirosaki-u.ac.jp 